Calculation of the absorbed dose distribution due to irregularly shaped photon beams usin... Page 1 of 2

\title{
IOPScience
}

\author{
Physics in Medicine and Biology $>$ Volume $41>$ Number 4
}

Pascal Storchi and Evert Woudstra 1996 Phys. Med. Biol. 41637 doi:10.1088/0031-9155/41/4/005

(http://dx.doi.org/10.1088/0031-9155/41/4/005)

\section{Calculation of the absorbed dose distribution due to irregularly shaped photon beams using pencil beam kernels derived from basic beam data}

Pascal Storchi and Evert Woudstra

Show affiliations

Dr Daniel den Hoed Cancer Centre, Groene Hilledijk 301, PO Box 5201, 3008 AE Rotterdam, The Netherlands

\section{AbstractMetrics}

In radiotherapy, accurately calculated dose distributions of irregularly shaped photon beams are needed. In this paper, an algorithm is presented which enables the calculation of dose distributions due to irregular fields using pencil beam kernels derived from simple basic beam data usually measured on treatment units, i.e. central axis depth - dose curves and profiles. The only extra data that are needed, and are not currently measured, is the phantom scatter factor curve at the reference depth. The algorithm has been developed as an extension to a previously developed algorithm for rectangular fields which is based on the Milan - Bentley storage model. In the case of an irregular field, the depth dose and the boundary function are computed by convolution of a field intensity function with pencil beam kernels. The depth dose is computed by using a 'scatter' kernel, which is derived from the stored depth - dose curves and from the phantom scatter factor curve. The boundary function is computed by using a 'boundary' kernel, which is derived from the boundary profile of a number of large square fields. Because of the simplicity of the data used and the underlying concepts, which for instance do not separate the head scatter from the primary beam, this algorithm presents some shortcomings. On the other hand, this simplicity is also of great advantage and the inaccuracy is acceptable for most clinical situations.

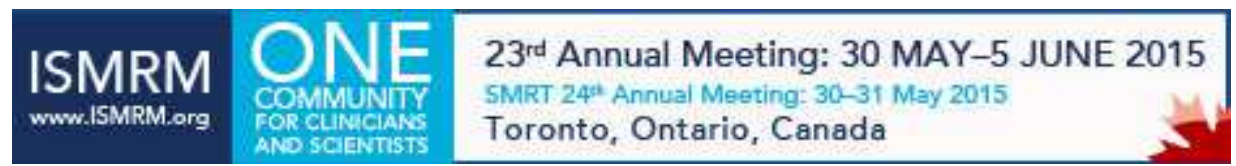

(http://oas.iop.org/5c/iopscience.iop.org/L18/813458094/Middle/IOPP/IOPs-Mid-PMB-ISMRM15Mar15/NEW-IOP-AD.468.jpg/676e4f6e3856566c615234414359386b?x)

PACS 
Calculation of the absorbed dose distribution due to irregularly shaped photon beams usin... Page 2 of 2

87.53.Bn Dosimetry/exposure assessment

87.55.-x Treatment strategy

Subjects

Medical physics

Dates

Issue 4 (April 1996)

Received 30 June 1995, in final form 19 January 1996

Metrics

Total article downloads: $\mathbf{3 7 5}$

More metrics

Permissions

Get permission to re-use this article (http://www.copyright.com/ccc/openurl.do?\&issn=13616560\&WT.mc.id $=$ )

\section{IOP Publishing}

(C) 2015 IOP Publishing 